\title{
Bacteroides are associated with GALT iNKT cell function and reduction of microbial translocation in HIV-1 infection
}

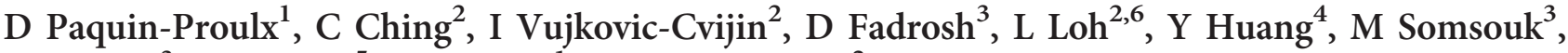 \\ SV Lynch ${ }^{3}$, PW Hunt ${ }^{5}$, DF Nixon ${ }^{1}$ and D SenGupta ${ }^{2}$
}

Invariant natural killer T (iNKT) cells are innate-like T cells that respond to lipid antigens presented by CD1d. These immunoregulatory cells have the capacity for rapid cytokine release after antigen recognition and are essential for the activation of multiple arms of the immune response. HIV-1 infection is associated with iNKT cell depletion in the peripheral blood; however, their role in the gastrointestinal-associated lymphoid tissue (GALT) is less well studied. Our results show that iNKT cells are found at a higher frequency in GALT compared with blood, particularly in HIV-1 elite controllers. The capacity of iNKT cells to produce interleukin-4 (IL-4) and IL-10 in the GALT was associated with less immune activation and lower markers of microbial translocation, whereas regulatory $T$ cell frequency showed positive associations with immune activation. We hypothesized that the composition of the microbiota would influence iNKT cell frequency and function. We found positive associations between the abundance of several Bacteroides species and iNKT cell frequency and their capacity to produce IL-4 in the GALT but not in the blood. Overall, our results are consistent with the hypothesis that GALT iNKT cells, influenced by certain bacterial species, may have a key role in regulating immune activation in HIV-1 infection.

\section{INTRODUCTION}

HIV-1 infection leads to the development of chronic inflammation that persists even in antiretroviral therapy (ART)treated individuals with undetectable viral loads. ${ }^{1,2}$ This inflammation is associated with non-HIV comorbidities, including cardiovascular disease, neurologic disorders, cancers, and an overall increased mortality. It has become apparent that immune activation is a better predictor of HIV-1 disease progression than either peripheral blood $\mathrm{CD} 4{ }^{+} \mathrm{T}$-cell count or viral load, ${ }^{3}$ highlighting the importance of chronic immune activation. However, distinct pathways of immune activation (innate vs. adaptive) appear to have differential prognostic capacity, depending on the cohorts. ${ }^{4}$ Importantly, while ART significantly diminishes immune activation (particularly if initiated early after infection ${ }^{5}$ ), the levels do not normalize to those of uninfected individuals. Invariant natural killer $\mathrm{T}$ (iNKT) cells are innate-like $\mathrm{T}$ cells that respond to lipid antigens presented on $\mathrm{CD} 1 \mathrm{~d}$, an major histocompatibility complex class I-like molecule expressed on antigen-presenting cells (APCs). ${ }^{6}$ iNKT cells are characterized by their expression of the semi-invariant $\mathrm{T}$-cell receptor chain V $\alpha 24-\mathrm{J} \alpha 18$ preferentially paired to a V $\beta 11$ chain. Upon stimulation, iNKT cells are capable of rapid production of a vast array of cytokines and chemokines and are instrumental in orchestrating innate and adaptive immune response. ${ }^{7}$ iNKT cells can recruit and modulate other immune cells, including natural killer (NK) cells, dendritic cells (DCs), and conventional CD4 ${ }^{+}$and CD8 ${ }^{+}$ T cells. ${ }^{8}$ Depending on the type of specific interactions between

${ }^{1}$ Department of Microbiology, Immunology and Tropical Medicine, The George Washington University, Washington, District of Columbia, USA. ${ }^{2}$ Division of Experimental Medicine, Department of Medicine, University of California, San Francisco, San Francisco, California, USA. ${ }^{3}$ Division of Gastroenterology, Department of Medicine, University of California, San Francisco, San Francisco, California, USA. ${ }^{4}$ Department of Bioengineering and Therapeutic Sciences, School of Pharmacy University of California, San Francisco, California, USA and ${ }^{5}$ HIV/AIDS Division, Department of Medicine, San Francisco General Hospital, University of California, San Francisco, San Francisco, California, USA. Correspondence: D Paquin-Proulx (dpaquin_proulx@gwu.edu)

${ }^{6}$ Current address: Department of Microbiology and Immunology, University of Melbourne, at the Peter Doherty Institute for Infection and Immunity, Melbourne, Victoria, Australia (L.L.). 
iNKT cells and DCs, the cytokines secreted by activated iNKT cells may either activate or suppress adaptive immune responses.

Mouse studies have shown that the symbiotic microbiota can impact the maturation and function of iNKT cells in the mucosa., ${ }^{9,10}$ A sphingolipid produced by the human commensal Bacteroides fragilis has been shown to bind CD1d and modulate iNKT cells. ${ }^{11}$ When compared with specific pathogen-free mice, germ-free mice have a greater frequency of iNKT cells in intestinal lamina propria and epithelium, but these cells express lower levels of activation and produce less cytokines in response to stimulation. ${ }^{12}$ Therefore, the gut microbiome influences the post-thymic maturation of iNKT cells, and intestinal bacterial reconstitution is a potential strategy for correcting systemic iNKT hyporesponsiveness in individuals with an altered microbial landscape. Dysbiosis of gut microbiota, particularly depletion of Bacteroidia members (including $B$. fragilis), has recently been described in the context of untreated HIV-1 infection, and is associated with markers of systemic immune activation and chronic inflammation. ${ }^{13}$

Mouse studies have revealed a role for iNKT cells in the control of viral infections, but their involvement in viral immunity in humans is less well characterized. ${ }^{14,15}$ Previous studies have shown that iNKT cells in the peripheral blood are selectively and rapidly depleted in early HIV-1 infection ${ }^{16}$ and in models of Simian immunodeficiency virus (SIV)-infected non-human primates. ${ }^{17}$ Some studies reported reduced iNKT proliferation and cytokine secretion (interferon- $\gamma$ (IFN $\gamma$ ), tumor necrosis factor (TNF), and IL-4 in response to $\alpha$ Gal-Cer/IL-2/PMA (phorbol 12-myristate 13-acetate) stimulation in HIV-1 infection, with variable restoration of function on antiretroviral therapy (ART). ${ }^{18-20}$ The role of iNKT cells in HIV-1 progression, whether defined by viral replication or immune activation, is unclear. Furthermore, HIV-1 has evolved to escape direct recognition of infected cells by iNKT cells (Paquin-Proulx et al, unpublished observation). Given their ability to produce IL-10 and activate regulatory T cells (Tregs), iNKT cells have the potential to help control pathologic T-cell activation. $^{21}$ In the present study, we investigated the role of peripheral blood and gut iNKT cells in controlling immune activation in HIV-1 infection and the consequences of gut microbial dysbiosis on iNKT frequency and function.

\section{RESULTS}

iNKT cells are reduced in the blood but not in the GALT during HIV-1 infection

A total of $23 \mathrm{HIV}$-1-infected subjects and 10 healthy controls were enrolled in the study and paired blood and gut-associated lymphoid tissue (GALT) samples were obtained (Table 1). Thirteen of the HIV-infected subjects were on ART at the time of sampling, and 3 of the 10 untreated patients met the definition of viremic controllers (viral load below 200 copies per $\mathrm{ml}$ ). Mononuclear cells were isolated from the samples and flow cytometry was performed. Staining with V $\alpha 24$, together with PBS57-CD1d tetramer, was used to identify iNKT cells (Figure 1a). iNKT cells were increased in the GALT compared with the blood across all subjects (Supplementary Figure 1A online). As reported previously, iNKT cells were found at a reduced frequency in the blood of viremic and ART-treated HIV subjects compared with healthy controls (Figure 1b). ${ }^{22-24}$ There was a trend for increased iNKT frequency in the ART-treated group compared with the viremic group $(P=0.07)$. Surprisingly, no change in iNKT cell frequency was observed in the GALT of viremic and ART-treated HIV-infected individuals compared with healthy controls (Figure 1c). HIV controllers appeared to have preserved iNKT cell frequency in the blood and higher frequency in the GALT compared with all other groups. Next, we investigated the distribution of the CD4+ subset of iNKT cells as this population has been shown to be preferentially depleted in the peripheral blood during HIV infection. ${ }^{22,23}$ No significant differences were observed between HIV-infected individuals and controls (Figure 1d) and between the viremic and ARTtreated groups (Supplementary Figure 1B,C) both in the blood and in GALT. However, HIV-infected subjects had a significantly increased proportion of CD4 + iNKT cells in GALT compared with the blood (Figure 1d). Our results confirm the loss of iNKT cell in the blood of HIV-infected individuals that has been reported by several studies before and suggest for the first time that iNKT cells may be preserved in the GALT in these patients.

\section{iNKT cells in the GALT of HIV-infected individuals have a Th2 cytokine profile}

Previous studies demonstrated that cytokine production by iNKT in the blood of HIV-infected subjects is impaired. ${ }^{19}$ However, cytokine production by iNKT cells in the GALT may be more relevant during HIV-1 disease progression. To assess

Table 1 Subjects demographics

\begin{tabular}{|c|c|c|c|c|c|c|}
\hline & Gender & Age (years) & CD4 count & Viral load & $\begin{array}{c}\text { Duration of infection } \\
\text { (years) }\end{array}$ & $\begin{array}{l}\text { Time on ART } \\
\text { (total years) }\end{array}$ \\
\hline Healthy $(n=10)$ & $9 \mathrm{M}, 1 \mathrm{~F}$ & $32.5(23-59)$ & $828(538-1,173)$ & & & \\
\hline Viremic $(n=7)$ & $5 \mathrm{M}, 1 \mathrm{M}$ to $\mathrm{F}, 1 \mathrm{~F}$ & $46(31-61)$ & $458(257-887)$ & $13,187(1,102-305,178)$ & $6.0(0-23)$ & $0(0-0.97)$ \\
\hline $\operatorname{ART}(n=13)$ & $12 \mathrm{M}, 1 \mathrm{~F}$ & $54(32-66)$ & $616(374-1,023)$ & Undetectable & $24.5(1-34)$ & $7.9(0.9-19.6)$ \\
\hline
\end{tabular}

Abbreviations: ART, antiretroviral therapy; F, female; M, male. 
a

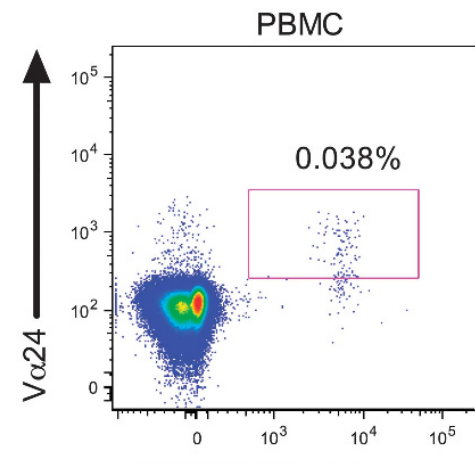

b

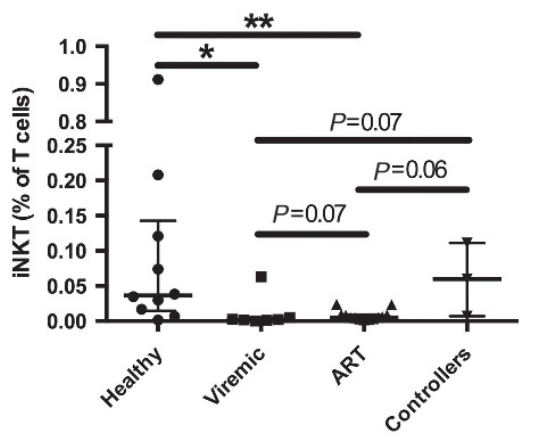

GALT

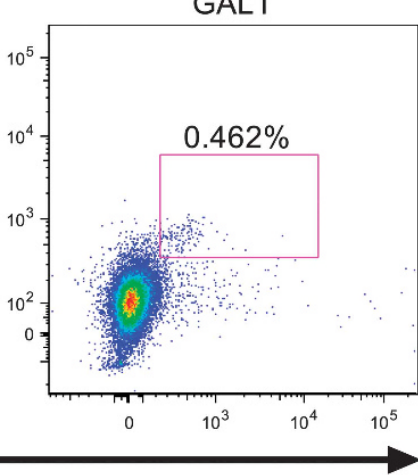

C

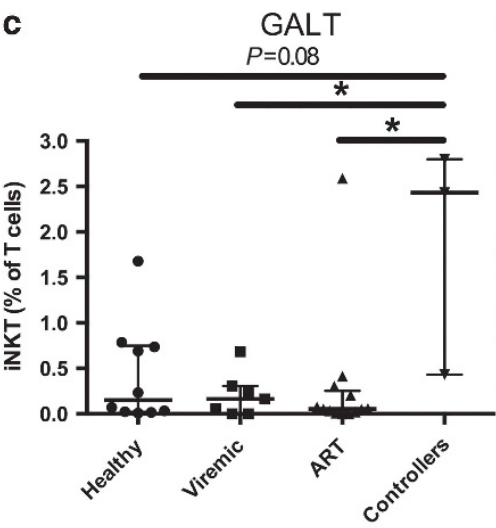

d

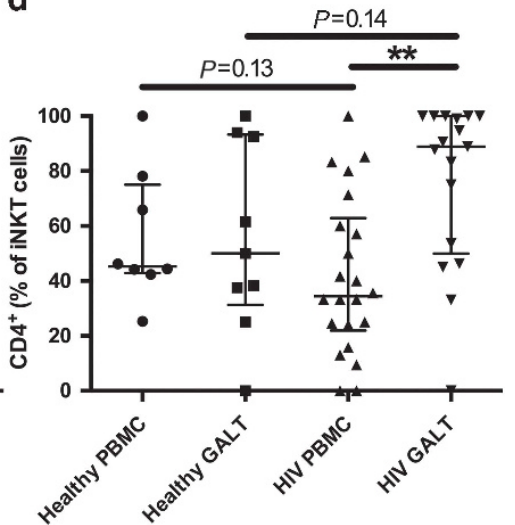

Figure 1 Frequency of invariant natural killer T (iNKT) cells in the blood and gastrointestinal-associated lymphoid tissue (GALT). (a) Representative gating strategy, iNKT cells were identified based on $V \alpha 24$ and PBS57-CD1d tetramer-positive stainings. Frequency of iNKT cells in the (b) blood and in the (c) GALT for healthy controls $(n=10)$, viremic $(n=7)$, antiretroviral therapy (ART)-treated $(n=13)$, and viremic controllers HIV-infected subjects $(n=3)$. (d) Frequency of iNKT cells expressing CD4 in the blood and GALT of healthy controls (blood, $n=8$ and GALT, $n=9$ ) and HIV-infected subjects (blood, $n=22$ and GALT, $n=17$ ). ${ }^{\star} P<0.05$ and ${ }^{* \star} P<0.01$. PBMC, peripheral blood mononuclear cell.

a

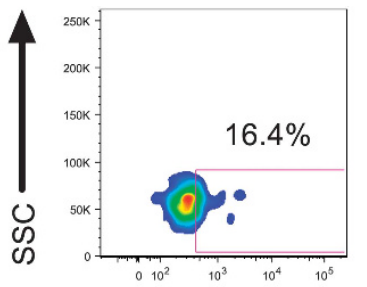

$\mathrm{IFN} \gamma$

b

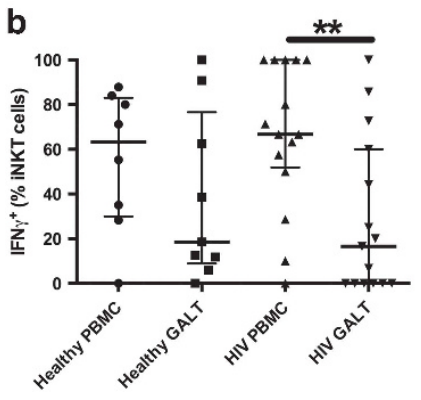

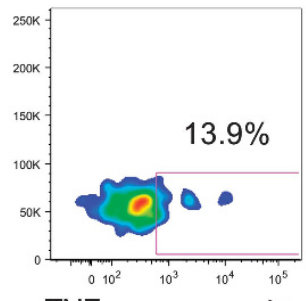

TNF

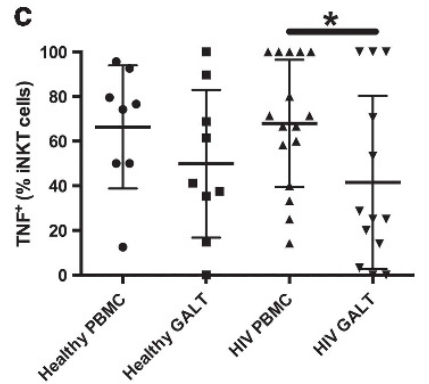

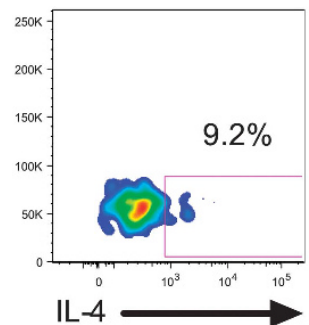
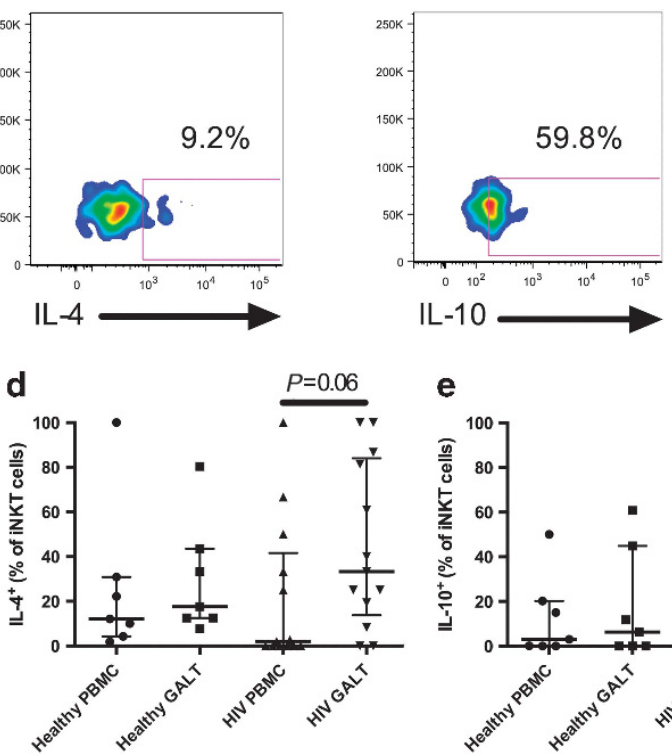

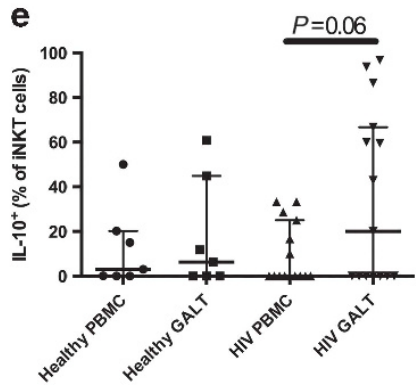

Figure 2 Cytokine production by invariant natural killer T (iNKT) cells in the blood and gastrointestinal-associated lymphoid tissue (GALT). Cells were stimulated with phorbol 12-myristate 13-acetate (PMA) and ionomycin before intracellular staining for cytokines. (a) The gates were set using unstimulated controls, representative staining for interferon- $\gamma$ (IFN $\gamma$ ), tumor necrosis factor (TNF), interleukin-4 (IL-4) and IL-10. Percentage of iNKT cells producing (b) IFN $\gamma$ (controls: blood, $n=8$ and GALT, $n=9$ and HIV-infected subjects: blood, $n=16$ and GALT, $n=15$ ), (c) TNF (controls: blood, $n=8$ and GALT, $n=9$ and HIV-infected subjects: blood, $n=16$ and GALT, $n=13$ ), (d) IL-4 (controls: blood and GALT, $n=7$ and HIV-infected subjects: blood and GALT, $n=13$ ) and (e) IL-10 (controls: blood and GALT, $n=7$ and HIV-infected subjects: blood and GALT, $n=15$ ). ${ }^{*} P<0.05$ and ${ }^{\star \star} P<0.01$. PBMC, peripheral blood mononuclear cell; SSC, side scatter. 
the functional potential of iNKT cells, peripheral blood mononuclear cell (PBMC) and rectal mononuclear cells were stimulated with PMA and ionomycin and the production of IFN $\gamma$, TNF, IL-4, and IL-10 was evaluated by flow cytometry (Figure 2a). No differences were observed in cytokine production by iNKT cells in the blood and GALT of HIVindividuals compared with healthy controls. The majority of iNKT cells in the blood of healthy controls produced IFN $\gamma$ and TNF, while the frequencies of IL-4- and IL-10-producing iNKT were low (Figure $\mathbf{2 b}-\mathbf{e}$ ). However, we observed a trend for a lower frequency of IFN $\gamma+$ and TNF + iNKT cells in the GALT of healthy individuals compared with the blood. This difference was even more marked in the HIV-infected subjects. The percentage of iNKT in the GALT-producing IL-4 and IL-10 varied greatly, ranging from undetectable to $100 \%$. There was a trend for higher IL- $4+(P=0.06)$ and IL-10 $+(P=0.06)$ iNKT cells in the GALT compared with the blood for HIVinfected individuals only. Our results suggest that a greater proportion of GALT iNKT cells in HIV subjects have a Th2 cytokine profile compared with the blood.

\section{Production of IL-4 and IL-10 by iNKT is associated with lower immune activation in the blood of HIV-infected subjects}

Next, we evaluated immune activation of $\mathrm{CD} 4{ }^{+}$and $\mathrm{CD} 8{ }^{+} \mathrm{T}$ cells in the blood and in the GALT by measuring coexpression of CD38 and HLA-DR. In healthy controls, we found a trend towards increased activation of $\mathrm{CD}^{+}{ }^{+} \mathrm{T}$ cells in the GALT compared with the blood (Figures 3a, $P=0.08$ ). Significantly higher levels of CD38 and HLA-DR coexpression were found
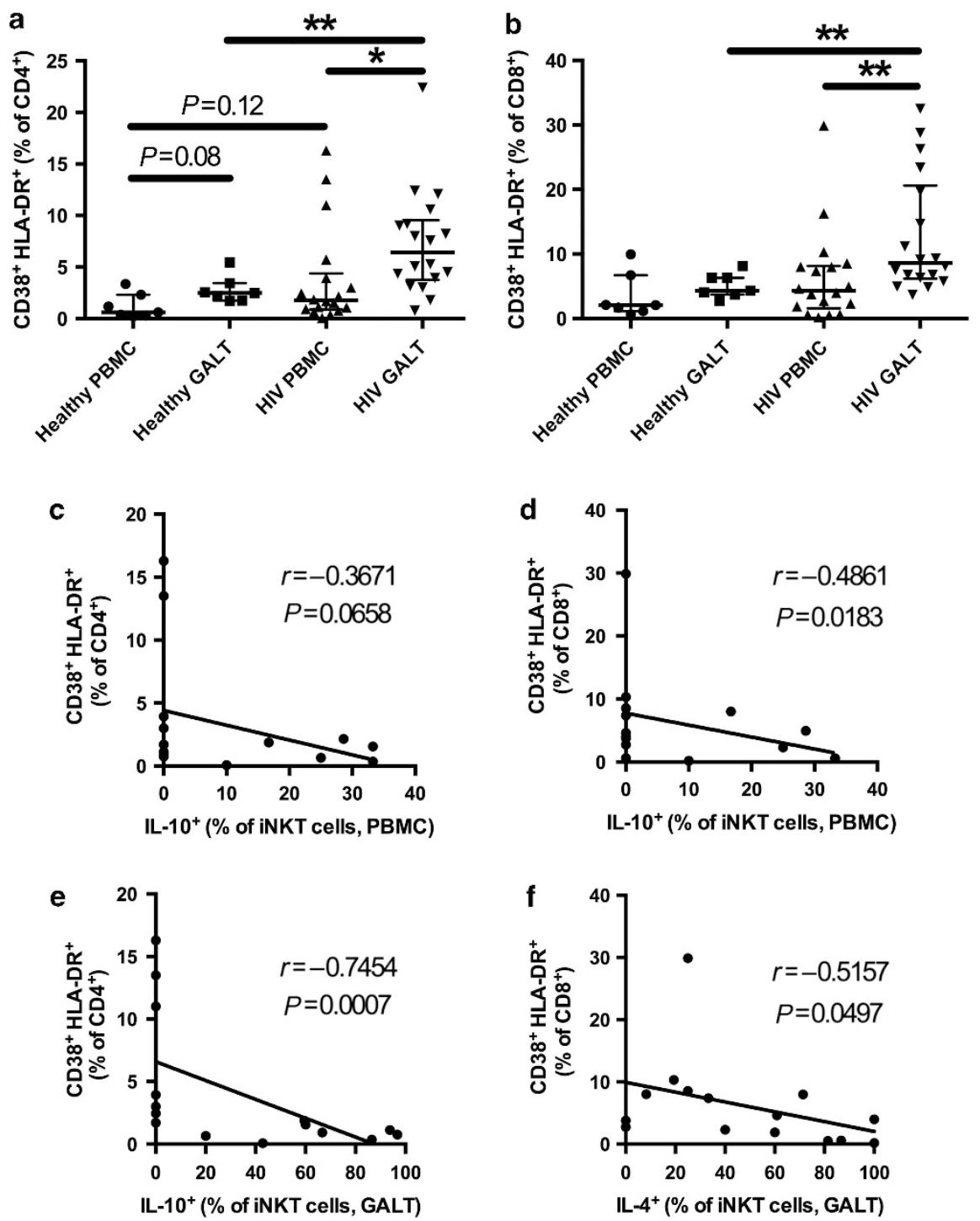

Figure $3 \mathrm{CD}^{+}$and $\mathrm{CD}^{+}$T-cell activation in the blood and gastrointestinal-associated lymphoid tissue (GALT) and associations with invariant natural killer T (iNKT) cell cytokine production. Coexpression of CD38 and HLA-DR on (a) CD4 ${ }^{+} \mathrm{T}_{\text {cells }}$ and (b) $\mathrm{CD} 8^{+} \mathrm{T}^{+}$cells in the blood and GALT of controls $(n=7)$ and HIV-infected subjects $(n=18)$. Associations between interleukin-10-positive (IL-10 $\left.{ }^{+}\right)$iNKT cells in the (c) blood and

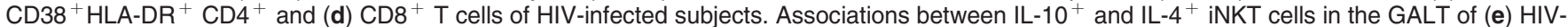
infected subjects and $\mathrm{CD} 38^{+} \mathrm{HLA}-\mathrm{DR}{ }^{+} \mathrm{CD} 4^{+}$and (f) $\mathrm{CD} 8^{+}$T cells, respectively. ${ }^{*} P<0.05$ and ${ }^{* \star} P<0.01$. PBMC, peripheral blood mononuclear cell. 
on $\mathrm{CD}^{+}$and $\mathrm{CD} 8^{+} \mathrm{T}$ cells in the GALT of HIV-infected individuals when compared with paired blood samples (Figure 3a,b). A nonsignificant trend for greater cellular activation in the blood was observed when comparing HIVinfected subjects to controls. However, HIV-infected individuals had significantly increased activation of both $\mathrm{CD}^{+}$and $\mathrm{CD}^{+}{ }^{+} \mathrm{T}$ cells in the GALT compared with healthy controls. Single expression of CD38 was also analyzed (Supplementary Figure 3A,B). iNKT cells are believed to have regulatory functions, which are in part mediated by their capacity to produce cytokines. ${ }^{7}$ Therefore, we investigated if there was any association between cytokine production by iNKT cells and immune activation in HIV-infected subjects. We found no associations between immune activation in the GALT and iNKT cell cytokine production (data not shown). However, IL-10 production by iNKT cells in the blood was associated with lower $\mathrm{CD} 4^{+}$and $\mathrm{CD} 8{ }^{+} \mathrm{T}$-cell activation in the blood (Figure 3c,d). Furthermore, IL-10 and IL-4 production by GALT iNKT cells were, respectively, associated with lower $\mathrm{CD}^{+}$and $\mathrm{CD} 8^{+}$T-cell immune activation in the blood (Figure 3e,f). HIV-infected subjects were then grouped according to the capacity of iNKT cells to produce IL-10 and the levels of immune activation were compared. Subjects with GALT iNKT cells producing IL-10 had significantly lower frequencies of activated peripheral $\mathrm{CD}^{+} \quad \mathrm{T}$ cells (Supplementary Figure 2A) and subjects with blood iNKT cells producing IL-10 showed a trend for lower levels of activated peripheral $\mathrm{CD} 4{ }^{+}$and $\mathrm{CD} 8{ }^{+} \mathrm{T}$ cells (Supplementary Figure 2B, C). In addition, we analyzed CD38 single expression and its associations with iNKT cell cytokine production. CD38 expression was inversely associated with IL-4 and IL-10 production by GALT iNKT cells and IL-10 production by blood iNKT cells (Supplementary Figure 3). These results suggest a role for GALT iNKT cells in dampening the pathological peripheral immune activation in HIV-1 infection.

In addition to iNKT cells, Tregs are also known to have an important role in modulating immune activation. ${ }^{25}$ Therefore, we analyzed Tregs (defined as $\mathrm{CD}^{+}, \mathrm{CD}^{+}, \mathrm{CD} 25^{+}$, and Foxp $3^{+}$) frequencies in peripheral blood and GALT of HIVinfected individuals. Similar frequencies of Tregs across groups were observed in the blood and in the GALT (Supplementary Figure 4A) and no difference in the frequency of Tregs in the blood compared with the GALT was observed for controls and HIV-infected individuals. We observed a positive association between Treg frequency in the GALT and $\mathrm{CD} 4^{+}$and CD8 ${ }^{+}$ T-cell activation in the GALT of HIV-infected individuals (Supplementary Figure 4B,C). No associations were found between Treg and iNKT cell frequency or function (data not shown).

\section{Cytokine production by GALT iNKT cells is associated with lower microbial translocation}

Damage to the integrity of the gut epithelial barrier by HIV-1 infection has been reported to lead to the presence of microbial products in the circulation, often referred to as microbial translocation (MT). MT has been associated with the pathologic immune activation that is characteristic of HIV1 disease. ${ }^{26}$ The kynurenine (Kyn) pathway of tryptophan (Trp) catabolism has been demonstrated to be dysregulated in HIV-1 infection, leading to an elevated Kyn/Trp ratio in the blood. ${ }^{27}$ This dysregulation is reported to be associated with changes in the composition of the microbiome of HIV-infected individuals and with established markers of disease progression such as IL$6^{13}$. We postulated that the immunoregulatory activity of GALT iNKT cells may limit disturbance to the gut barrier and therefore lower immune activation by reducing MT. For this purpose, we measured the concentration of soluble CD14 (sCD14) and LPS-binding protein (LBP) in the plasma as they have been shown to be indirect markers of MT. ${ }^{26,28}$ We also measured intestinal fatty acid-binding protein 2 (, a marker of gut damage ${ }^{29}$ ), IL-6, and the Kyn/Trp ratio. As expected, HIVinfected subjects had significantly elevated levels of sCD14, LBP, and intestinal fatty acid-binding protein 2 (Figure $4 a-c$ ), suggesting gut epithelial barrier dysfunction and MT. The Kyn/ Trp ratio was also elevated in HIV-infected individuals (Figure 4d) as well as the levels of IL-6 (Supplementary Figure 5). We then looked for relationships between all of the above parameters and iNKT frequency or function. The capacity of peripheral iNKT cells to produce IL-4 was positively associated with plasma levels of sCD14 and there was a trend for an inverse association between the capacity of intestinal iNKT cells to produce IL-10 and the levels of sCD14 (Figure 4e,f). iNKT cell production of IL-4 and TNF- $\alpha$ in the GALT showed negative associations with the levels of LBP (Figure $4 \mathbf{g}, \mathbf{h}$ ). Finally, IL-4 production by peripheral iNKT cells was associated with elevated Kyn/Trp ratios (Figure 4i). Additionally, HIV-infected subjects were grouped according to capacity of iNKT cells to produce IL-4 or IL-10. We observed that individuals in the group with higher production of IL-4 had lower levels of sCD14 (Supplementary Figure 6). Taken together, our results show that a higher capacity to produce cytokines by iNKT in the GALT is associated with lower markers of MT, suggesting a role for GALT iNKT cells in modulating this pathological process in HIV-1 infection.

\section{Bacteroides are associated with iNKT frequency and IL-4 production in the GALT}

The composition of the bacterial gut microbiota of untreated HIVinfected subjects is distinct from that of healthy individuals, with ART-treated patients having an intermediate change in the microbiome profile. ${ }^{30}$ One of the genera significantly depleted in HIV-infected individuals is Bacteroides. We confirmed that Bacteroides were reduced in ART-treated HIV-infected subjects in our study (Figure 5a). Next, we performed an unbiased analysis of all gut-resident operational taxonomic units (OTUs) abundances compared with GALT iNKT cell frequency in ART-treated subjects and found that negative correlations existed between GALT iNKT cell frequency and OTUs in the Prevotella genus (Benjamini-Hochberg $Q$-value $<0.15$; Supplementary file), a genus that has been shown to be increased in abundance in HIV-infected subject gut microbiomes and associated with elevated mucosal immune 

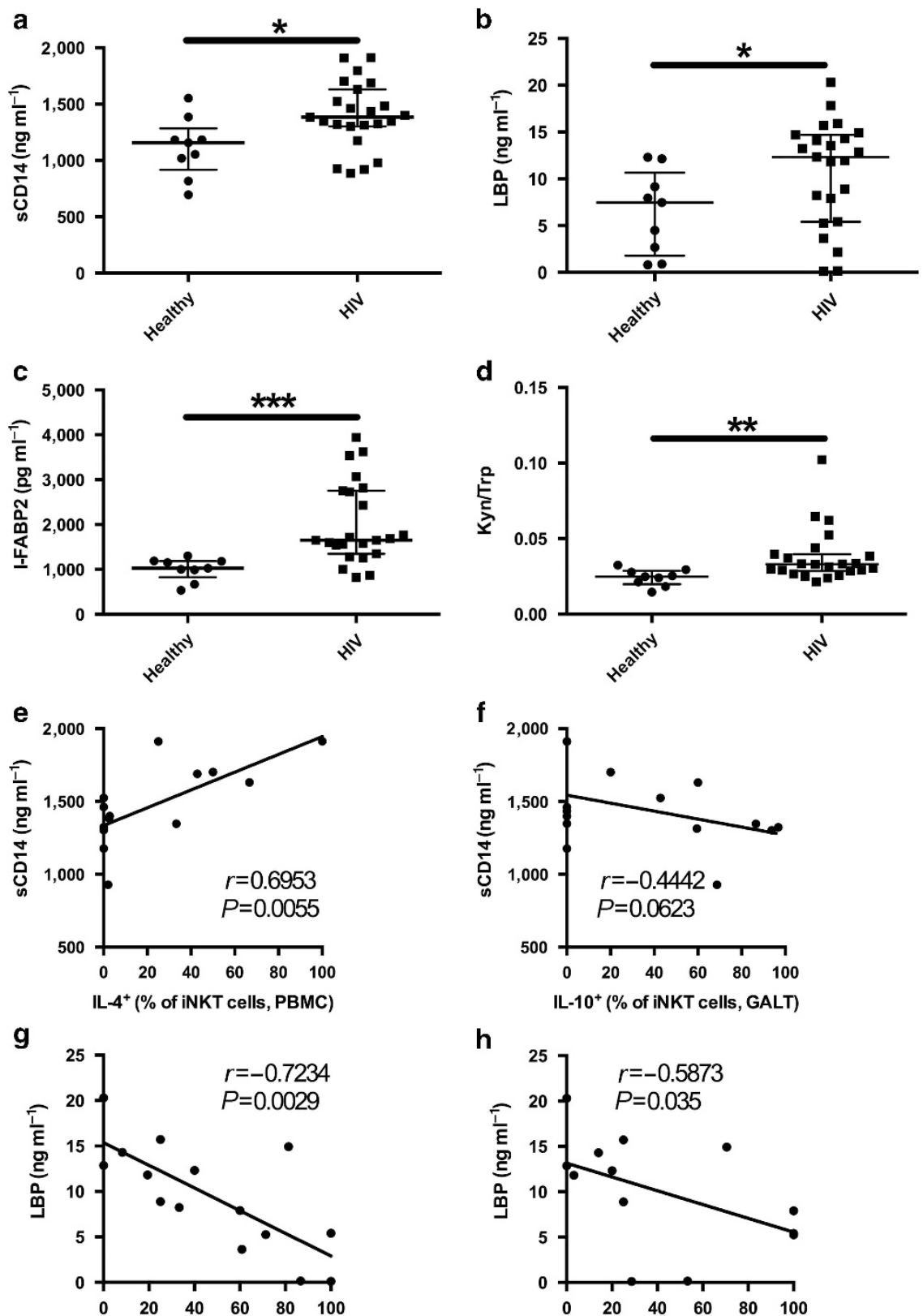

h
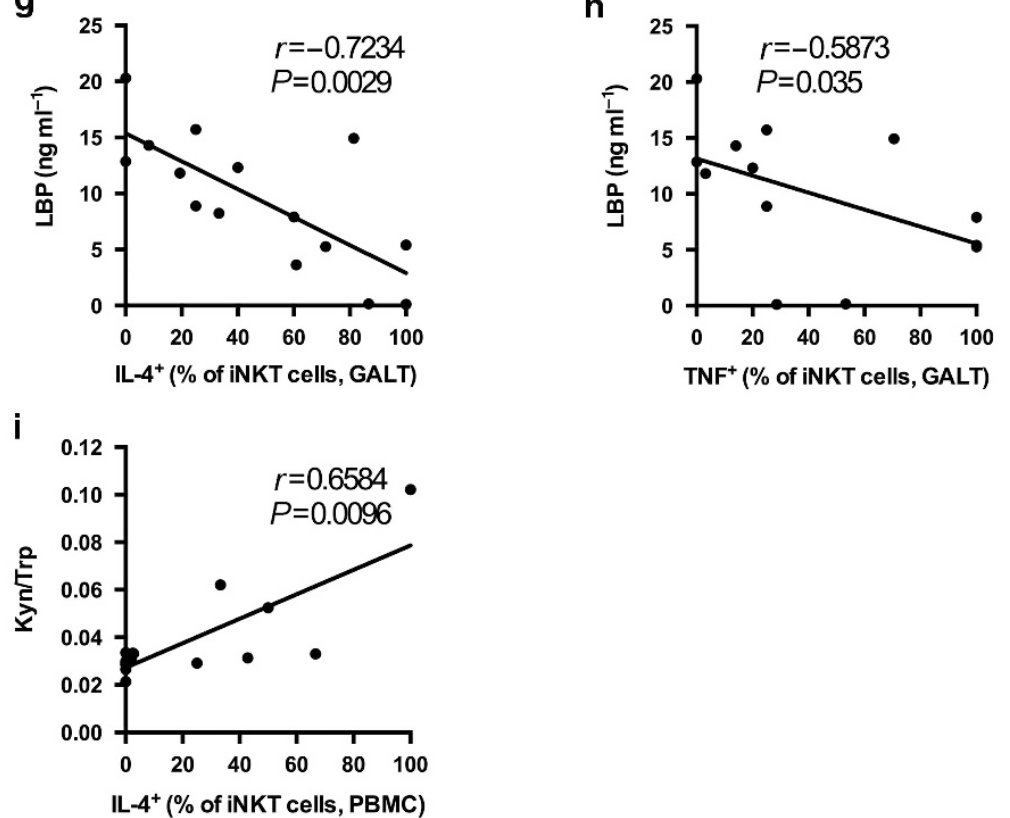

Figure 4 Markers of microbial translocation and associations with invariant natural killer T (iNKT) cell cytokine production. The levels of (a) SCD14, (b) LPS-binding protein (LBP), (c) intestinal fatty acid-binding protein 2 (I-FABP2), and (d) kynurenine/tryptophan (Kyn/Trp) ratio were determined in the serum of healthy controls $(n=9)$ and HIV-infected subjects $(n=23)$. Associations between the levels of sCD14 in HIV-infected subjects and IL4 ${ }^{+}$iNKT cells in the (e) blood and IL $10^{+}$iNKT cells in the (f) gastrointestinal-associated lymphoid tissue (GALT). Associations between LBP levels

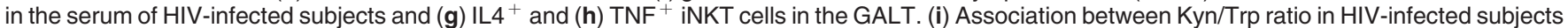
and IL4 ${ }^{+}$iNKT cells in the blood. ${ }^{*} P<0.05,{ }^{* *} P<0.01$ and ${ }^{* \star *} P<0.001$. PBMC, peripheral blood mononuclear cell. 
a

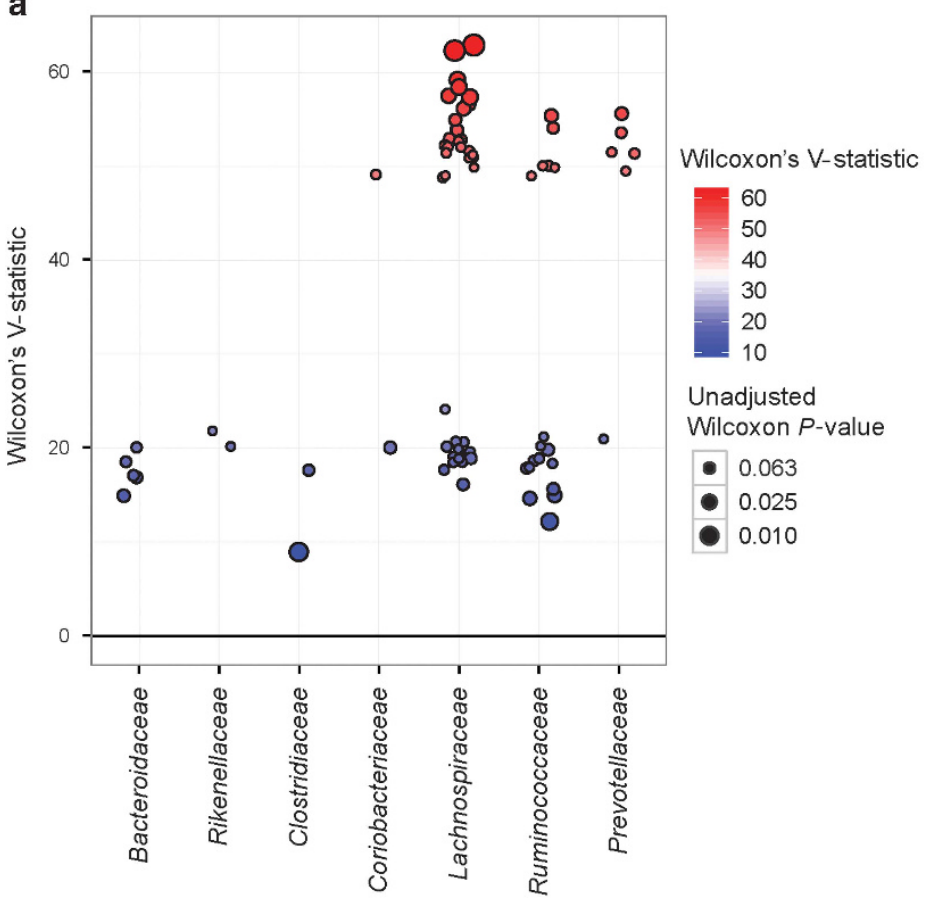

C

Bacteroides OTU correlations to GALT iNKT cell abundances (all OTUs, unadjusted Spearman $P<0.10$ )

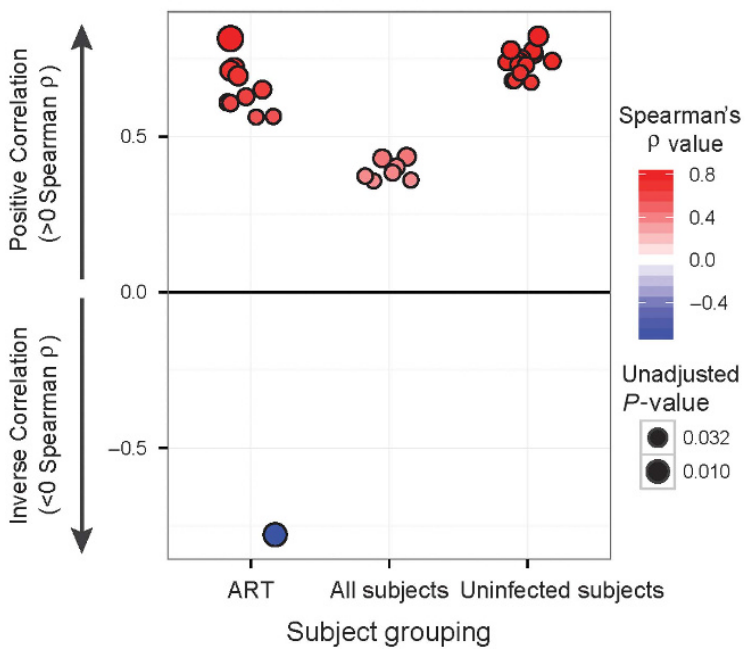

b

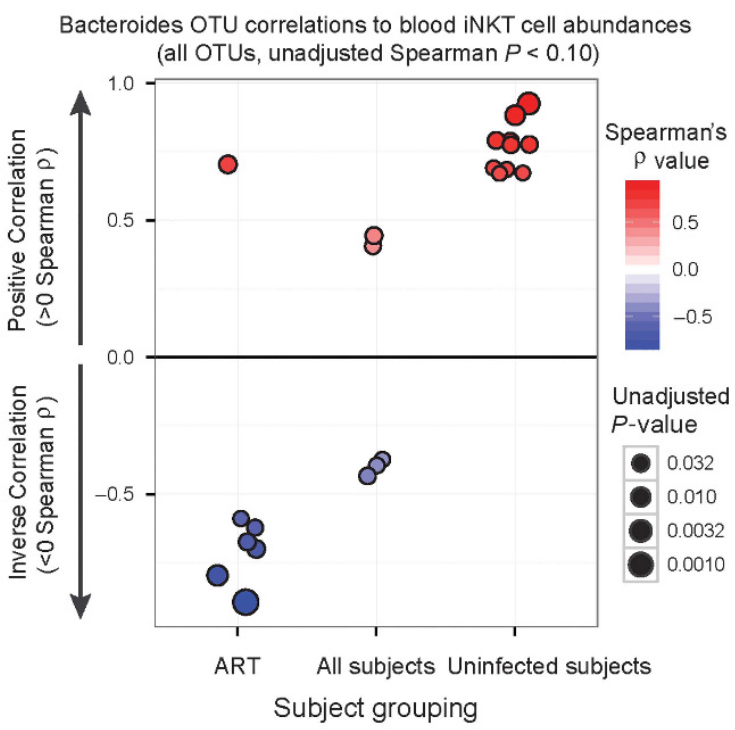

d

Bacteroides OTU correlations to percent IL-4+ GALT iNKT cells (all OTUs, unadjusted Spearman $P<0.10$ )

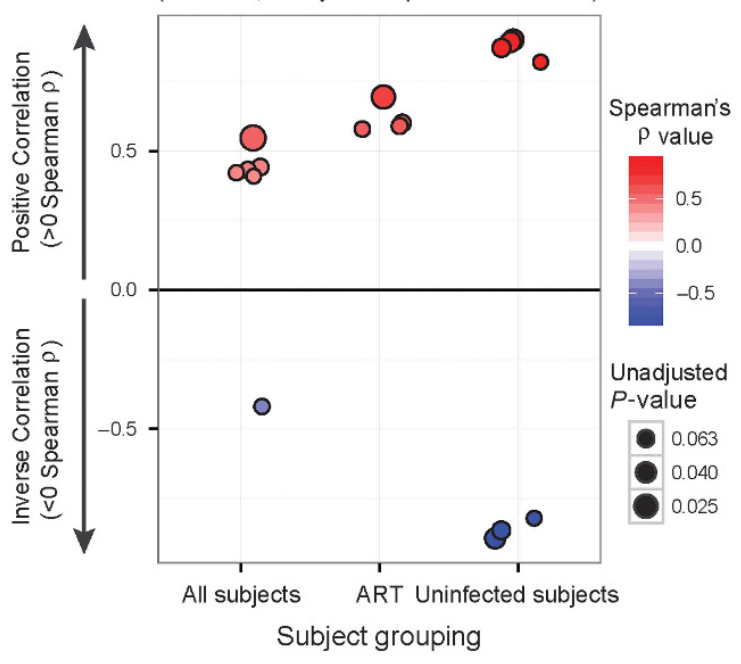

Figure 5 Change in microbiota in HIV-infected individuals and associations with invariant natural killer T (iNKT) frequency and function. Wilcoxon's rank-sum tests were performed comparing gut mucosal operational taxonomic unit (OTU) abundances between HIV-infected subjects undergoing antiretroviral (ART) and uninfected subjects. OTUs with $P<0.15$ are shown including taxonomic families to which each OTU belongs. (a) Wilcoxon's V-statistics (y axis) provide nonparametric enrichment/depletion information, and unadjusted $P$-values are depicted by point sizes. (b) Abundance of all detected OTUs within the Bacteroides genus were compared with blood iNKT cell percent abundances for three subject groups (ART HIV-infected, uninfected, and all subjects combined). (b) Spearman's $\rho$ values depict directionality of correlation, and all OTUs with $P<0.10$ shown. (c) Abundance of all Bacteroides OTUs were compared with gastrointestinal-associated lymphoid tissue (GALT) iNKT cell percent abundances for the same subject groups $\left(P<0.10\right.$ shown). Spearman's correlations were performed comparing all Bacteroides OTU relative abundances and proportions of IL-4 ${ }^{+}$ GALT iNKT cells following phorbol 12-myristate 13-acetate (PMA) and ionomycin stimulation $(P<0.10$ shown) (d).

activation. ${ }^{31}$ Given that $B$. fragilis expresses a glycolipid that can activate human iNKT cells, ${ }^{11}$ we investigated whether the abundance of OTUs within the Bacteroides genus was associated with frequencies of iNKT cells in both peripheral blood and GALT within ART-treated HIV-infected subjects, all study subjects combined, and uninfected subjects only (Figure $\mathbf{5 b , c}$ and Supplementary file). Fewer OTUs reached $P<0.10$ for comparisons to peripheral blood as opposed to GALT iNKT frequencies, and no trends were consistent across all subject groupings for comparisons to peripheral blood. However, when comparing OTU abundances to GALT iNKT frequencies, consistent positive associations were found between several Bacteroides OTUs and iNKT frequencies across subject groups. Finally, we looked for associations between Bacteroides OTUs and 
cytokine production by iNKT cells. We found a positive association between specific Bacteroides OTUs and the capacity of GALT iNKT cells to produce IL-4 (Figure 5d and Supplementary file), although these observations exhibited Benjamini-Hochberg false discovery rate $Q$ values $>0.70$. These results suggest that loss of the Bacteroides genus in HIV-infected individuals could influence both the frequency and function of iNKT cells in the gut.

\section{DISCUSSION}

Several studies have examined the frequency and function of peripheral blood iNKT cells in HIV-1 infection but limited information is available for the GALT, an important target in HIV-1 pathogenesis. We found that iNKT cells were depleted in the blood but not in the GALT of HIV-infected subjects and that GALT iNKT cells consisted of an increased proportion of the $\mathrm{CD}^{+}{ }^{+}$subset. This is in contrast to a previous study that reported that $\mathrm{CD}^{+}{ }^{+}$iNKT cells are lost in the gut of HIV-1-infected individuals. ${ }^{32}$ The discrepancy between our results and those of Ibarrondo et al. ${ }^{32}$ could be explained by important differences in the HIV-1 cohorts and the methods used to identify iNKT cells. The majority of our HIV-1-infected individuals had an undetectable viral load (ART-suppressed or controllers) and we used a CD1d tetramer to identify iNKT cells while the cohort of Ibarrondo et al. ${ }^{32}$ consisted exclusively of untreated subjects, and iNKT cells were identified using antibodies against the invariant T-cell receptor. Altogether, this would suggest that viral suppression prevents the depletion of the $\mathrm{CD} 4{ }^{+}$subset of iNKT cells in the GALT. Although based on a small number of subjects, our results suggest that HIV-1 elite controllers maintain normal iNKT cell frequency in the blood and have high levels of iNKT cells in the GALT. Further studies enrolling more controllers will be required to confirm the validity of these results.

$\mathrm{CD} 4{ }^{+}$iNKT are known to produce more Th2 cytokines than the $\mathrm{CD} 4{ }^{-}$subsets. ${ }^{33-35}$ Therefore, given our finding of higher percentages of $\mathrm{CD} 4{ }^{+}$iNKT in the gut as compared with blood in $\mathrm{HIV}$-infected subjects, it is not surprising that we observed more production of IL- 4 and IL-10, two Th2 cytokines, and less IFN $\gamma$ and TNF $\alpha$ by iNKT cells in the GALT compared with the blood. Our results suggest that iNKT cells can regulate the pathologic chronic immune activation in HIV-1 infection by their production of IL- 4 and IL-10 in the GALT as well as IL-10 in the blood. We could not detect iNKT cell production of IL-10 by a significant proportion of HIV-infected individuals, suggesting that they could be separated in two groups based on the capacity of iNKT cells to produce IL-10. However, this could be due to the detection limit of the assay. Further studies with more subjects in each group are required to resolve this matter. Our findings are consistent with the model proposed by Rout et al. ${ }^{36}$ who have shown that sooty mangabeys, natural hosts of SIV that do not progress to AIDS, exhibit a preservation of iNKT cell frequency and function after SIV infection. They have thus suggested that iNKT cell dysfunction has a role in AIDS pathogenesis. ${ }^{36}$ MT has been proposed as a major component driving immune activation in HIV-infected individuals. We found that production of IL-4, IL-10, and TNF by iNKT cells in the GALT, but not in the blood, were associated with lower MT as measured by sCD14 and LBP, suggesting that iNKT cell production of IL-10 in the blood could have a localized effect on immune activation while production of the same cytokine in the GALT could have a more generalized effect by reducing MT. In contrast, the frequency of Tregs in the GALT was positively associated with $\mathrm{CD}^{+}{ }^{+}$and $\mathrm{CD} 8^{+}$T-cell activation in the GALT. We saw no association between Tregs and markers of MT, suggesting a specific role for iNKT cells. How production of IL-4, IL-10, and TNF by GALT iNKT cells contributes to reduced MT remains to be determined. It is a likely possibility that the ability of iNKT cells to interact with antigen-presenting cells to shape the adaptive immune response may be involved. Moreover, mice lacking IL-10 have been shown to have increased gut permeability caused by an excessive Th1 response against enteric bacteria. ${ }^{37}$ On the other hand, the positive associations between IL-4 production by peripheral iNKT and Kyn/Trp ratio and sCD14 might represent in part their response to MT.

To our knowledge, this is the first study looking at the influence of the microbiome on iNKT cell frequency and function in humans. A study performed in mice showed that a sphingolipid derived from $B$. fragilis can inhibit iNKT cell activation and that $B$. fragilis colonization reduces the frequency of iNKT cells in the colon but not in other organs or in the blood. ${ }^{10}$ A different group reported that $B$. fragilis produced a sphingolipid that can activate iNKT cells. ${ }^{11}$ In our hands, multiple Bacteroides OTUs exhibited positive associations with GALT iNKT cells and only one OTU presented a negative association in $\mathrm{HIV}$-infected subjects (with the later association not being present in uninfected subjects). This would suggest that Bacteroides produce an antigen that can activate and expand iNKT cells in the GALT. The reported greater abundance of Bacteroides in HIV elite controllers compared with viremic patients ${ }^{38}$ could therefore contribute to the higher frequency of iNKT in the GALT of elite controllers in our study. Mouse studies have shown that animals kept in germ-free conditions have lower IFN $\gamma$, TNF, and IL-4 production by iNKT cells following stimulation with $\alpha$-GalCer, ${ }^{12}$ but the bacteria responsible for the functional maturation of iNKT cells were not identified. In this study, we have found a positive association between Bacteroides and IL-4 production by GALT iNKT cells. While fetal human iNKT cells have been reported to mature and acquire function in the small intestine before colonization by the normal microbiota, ${ }^{39}$ our results are consistent with the hypothesis that in adults the normal microbiota may provide signals that support GALT iNKT cell frequency and functionality. These findings would be strengthened by further study in larger human cohorts. V $\alpha 24^{-}$ cells specifically binding to $\alpha$-Gal-Cer-loaded CD1d tetramer have been detected in PBMC following in vitro expansion. ${ }^{40}$ However, the limited amount of GALT material obtained in our study did not allow us to study this population of NKT cells.

Based on our results, we propose a model where iNKT cells in the GALT have an important role in limiting MT and chronic pathologic immune activation in HIV-1 infection. This role of GALT iNKT is influenced by the composition of the gut 
microbiota, with loss of the Bacteroides genus in HIV-infected individuals, possibly affecting both iNKT frequency and function. This suggests that strategies boosting GALT iNKT cells could reduce the MT and persistent immune activation that are important factors in the morbidity caused by HIV-1.

\section{METHODS}

Study subjects. PBMCs and GALT samples were obtained from participants in the San Francisco-based HIV-1-infected SCOPE (Study on Cognition and Prognosis in the Elderly) cohort. Samples from HIV1 -seronegative controls were obtained from healthy volunteers. The study was approved by the local Institutional Review Board (University of California San Francisco Committee on Human Research), and individuals gave written informed consent. Samples were obtained from the following numbers and categories of HIV-1-infected individuals: 3 untreated virologic "controllers" (viral load, <200 HIV1 copies per ml), 13 HAART-suppressed patients (viral load, $<50$ copies per $\mathrm{ml}$ ), and 7 untreated "virologic noncontrollers" (viral load, $>1,000$ copies per $\mathrm{ml}$ ). All had CD4 ${ }^{+}$T-cell counts of $>250$ cells per $\mathrm{mm}^{3}$. See Table 1 for baseline subject characteristics.

Blood and GALT samples. Five milliliters of blood was collected in $\mathrm{BD}$ Vacutainer EDTA-coated tubes for PBMC and plasma isolation purposes. After centrifuging at $400 \mathrm{~g}$ for $10 \mathrm{~min}$ without braking, the plasma layer was removed and frozen at $-80^{\circ} \mathrm{C}$ for enzyme-linked immunosorbent assay quantification. The cellular fraction from the first spin was used to isolate PBMC by centrifugation over a FicollPaque (GE Healthcare, Uppsala, Sweden) layer at $800 \mathrm{~g}$ for $25 \mathrm{~min}$ without braking. The PBMC layer was then removed and washed two times in RPMI with L-glutamine, penicillin/streptomycin, HEPES, and $10 \%$ fetal bovine serum (referred now on as R-10) at $400 \mathrm{~g}$ for $10 \mathrm{~min}$ with braking. GALT from rectosigmoid biopsy specimens were placed on a shaking incubator at $37^{\circ} \mathrm{C}$ with a digestion mix of RPMI with + L-glutamine, HEPES, penicillin/streptomycin, and collagenase type II $\left(0.25 \mathrm{mg} \mathrm{ml}^{-1}\right)$ (Sigma-Aldrich, St Louis, MO). After one digestion of $30 \mathrm{~min}$, the sample was strained through a $70 \mu \mathrm{m}$ cell strainer and washed through with cold R-10. Undigested biopsies were transferred into the collagenase digestion mix for repeat digestion of $30 \mathrm{~min}$. Strained and digested biopsies were washed in R-10 and spun down at $700 \mathrm{~g}$ for $6 \mathrm{~min}$ at $4{ }^{\circ} \mathrm{C}$ to isolate the rectal mononuclear cell. PBMCs and rectal mononuclear cells were then resuspended and counted using Guava Viacount (Millipore, Brillerica, MA) on the Accuri C6 (BD Biosciences, San Jose, CA).

Flow cytometry and mAbs. For surface staining, cells were stained with surface markers for $30 \mathrm{~min}$ on ice and washed two times with FACS buffer (phosphate-buffered saline with $2 \%$ fetal bovine serum and $2 \mathrm{~mm}$ EDTA buffer). Later, cells were fixed and permeabilized with Fix/Perm buffer (BD Biosciences) for $20 \mathrm{~min}$ on ice, washed two times with BD Perm/Wash, and stained with the intracellular antibodies for $60 \mathrm{~min}$ on ice. For PoxP3 staining, cells were fixed and permeabilized with Fix/Perm Buffer (eBiosciences, San Diego, CA) for 60 min on ice, washed two times with Perm/Wash buffer (eBiosciences), and stained with intracellular antibodies for $60 \mathrm{~min}$ on ice. Subsequently, the cells were washed two times with the respective Perm/Wash buffer and kept in 2\% paraformaldehyde. Antibodies used: PBS57-CD1d tet antigen-presenting cell (kindly donated by NIH Tetramer Resource Facility, Emory University Vaccine Center, Atlanta, GA), V 224 FITC, and CD3 ECD were from Beckman Coulter (Fullerton, CA), CD4 Qdot655, CD8 Qdot 605, and the viability marker AmCyan were from Life Technologies (Carlsbad CA), CD25 APC, CD38 PE, HLA-DR PerCP, IFN $\gamma$ V450, TNF Alexa700, IL$10 \mathrm{PE}$, and IL-4 PE-Cy7 were all from BD Bioscience. Data were acquired on a BD LSRFortessa instrument (BD Biosciences) and analyzed using FlowJo Version 9.8.5 software (TreeStar, Ashland, OR).

Enzyme-linked immunosorbent assay. IL-6, sCD14, LBP, and FABP-2 levels were detected in plasma isolated from peripheral blood with a commercially available Enzyme-Linked Immunosorbent Assay (all from R\&D Systems, Minneapolis, MN) and performed according to the standard protocol. For sCD14 and IL-6, commercially available quality controls (R\&D Systems) were also performed to ensure accurate detection of the kits.

Microbiome sample processing and analysis. DNA was extracted from gut biopsy samples using the AllPrep DNA/RNA Mini Kit (Qiagen, Hilden, Germany) according to the manufacturer's recommendations. Each DNA sample was PCR amplified in triplicate using primer pairs that targeted the V4 hypervariable region of the $16 \mathrm{~S}$ rRNA gene, contained a unique barcode sequence to enable demultiplexing of pooled samples, and contained an adapter sequence that enables the amplicon to bind to the MiSeq flow cell. Successful amplicons were pooled in approximately equal molar concentrations and sequenced on the Illumina MiSeq platform. Paired sequencing reads were quality filtered and demultiplexed using the QIIME ${ }^{41}$ software package. Briefly, assembled sequencing read pairs were binned into OTUs (OTUs using a 97\% similarity to the Greengenes database) and reads that either did not cluster to the Greengenes database or that were chimeric were removed from subsequent analyses. OTUs that had a cumulative read count across all samples of $<0.001 \%$ of the total reads were removed from downstream analysis. Sample read numbers were rarefied to the read number of the lowest sample after processing $(94,780)$ resulting in a rarefied OTU table. OTU abundances were compared between uninfected and ART-treated HIV-infected subjects using a custom R script in conjunction with the "exactRankTests" package. Spearman's correlations were performed using the "Hmisc" R package using a custom script. Data visualizations were performed using the R package "ggplot2".

Trp metabolism. Liquid chromatography-tandem mass spectrometry was used to assess Kyn and Trp levels as described previously. ${ }^{42}$

Statistical analysis. All statistical analysis was performed using Graph Pad Prism version 6.0f for Mac OSX (GraphPad Software, La Jolla, CA). Groups were compared using the Mann-Whitney test, paired blood and GALT samples were compared using Wilcoxon's marched-pairs signedrank test. Associations between groups were determined by Spearman's rank correlation. $P$ values $<0.05$ were considered statistically significant.

SUPPLEMENTARY MATERIAL is linked to the online version of the paper at http://www.nature.com/mi

\section{ACKNOWLEDGMENTS}

This work was supported by NIAID (R01 Al52731), the Delaney AIDS Research Enterprise (DARE; Al096109), NIAID (K24 AI069994), the National Institutes of Health, University of California, San Francisco-Gladstone Institute of Virology and Immunology Center for AIDS Research, P30Al027763, the UCSF Clinical and Translational Research Institute Clinical Research Center (UL1 RR024131), the Center for AIDS Prevention Studies (P30 MH62246), the DC CFAR (P30Al117970 and P30AI087714), the Fundação de Amparo a Pesquisa do Estado de São Paulo (04/15856-9 and 2010/05845-0), CNPq/CAPES 056/2012, and the Peter and Shelagh Godsoe Family Foundation through the AIDS Research Institute at UCSF, and the CFAR Network of Integrated Systems (R24 AI067039). I.V.-C. was supported by CHRP D13-SF-388 and NSF 1144247. M.S. was supported by NIH NCI K23 CA157929. D.S. was supported by NIH NIAID K08 A120071. L.L. was supported by NH and MRC CJ Martin Fellowship. We acknowledge the NIH Tetramer Core Facility (contract HHSN272201300006C) for provision of CD1d tetramers. We thank Montha Pao, Monica Deswal, Rebecca Hoh, and Melissa Krone for SCOPE study coordination.

\section{AUTHOR CONTRIBUTIONS}

D.P.P. analyzed data and wrote the manuscript; C.C. performed experiments and helped write the manuscript; I.V.-C. analyzed microbiome data, helped write the manuscript, and helped obtain grant funding; D.F. performed microbiome profiling; L.L. helped with flow cytometry panels; Y.H. performed kynurenine/tryptophan assays; M.S. performed GALT biopsies; S.V.L. oversaw microbiome analyses; P.W.H. and D.F.N. gave input on overall study design, 
analysis, and helped write the manuscript; D.S. designed and obtained grant funding for the study, performed experiments and helped write the manuscript.

\section{DISCLOSURE}

The authors declared no conflict of interest.

Official journal of the Society for Mucosal Immunology

\section{REFERENCES}

1. Miedema, F. et al. Immune activation and collateral damage in AIDS pathogenesis. Front. Immunol. 4, 298 (2013).

2. Hatano, H. Immune activation and HIV persistence: considerations for novel therapeutic interventions. Curr. Opin. HIV AIDS 8, 211-216 (2013).

3. Liu, Z. et al. Elevated CD38 antigen expression on CD8 + Tcells is a stronger marker for the risk of chronic HIV disease progression to AIDS and death in the Multicenter AIDS Cohort Study than CD4 + cell count, soluble immune activation markers, or combinations of HLA-DR and CD38 expression. J. Acquir. Immune Defic. Syndr. Hum. Retrovirol. 16, 83-92 (1997).

4. Utay, N.S. \& Hunt, P.W. Role of immune activation in progression to AIDS. Curr. Opin. HIV AIDS 11, 131-137 (2016).

5. Jain, V. et al. Antiretroviral therapy initiated within 6 months of HIV infection is associated with lower T-cell activation and smaller HIV reservoir size. J. Infect. Dis. 208, 1202-1211 (2013).

6. Godfrey, D.I. et al. Antigen recognition by CD1d-restricted NKT T cell receptors. Semin. Immunol. 22, 61-67 (2010).

7. Matsuda, J.L., Mallevaey, T., Scott-Browne, J. \& Gapin, L. CD1d-restricted iNKT cells, the "Swiss-Army knife" of the immune system. Curr. Opin. Immunol. 20, 358-368 (2008).

8. Van Kaer, L., Parekh, V.V. \& Wu, L. Invariant natural killer T cells: bridging innate and adaptive immunity. Cell Tissue Res. 343, 43-55 (2011).

9. Olszak, T. et al. Microbial exposure during early life has persistent effects on natural killer T cell function. Science 336, 489-493 (2012).

10. An, D. et al. Sphingolipids from a symbiotic microbe regulate homeostasis of host intestinal natural killer T cells. Cell 156, 123-133 (2014).

11. Wieland Brown, L.C. et al. Production of alpha-galactosylceramide by a prominent member of the human gut microbiota. PLoS Biol. 11, e1001610 (2013).

12. Wingender, G. et al. Intestinal microbes affect phenotypes and functions of invariant natural killer T cells in mice. Gastroenterology 143, 418-428 (2012).

13. Vujkovic-Cvijin, I. et al. Dysbiosis of the gut microbiota is associated with HIV disease progression and tryptophan catabolism. Sci. Transl. Med. 5, 193ra191 (2013)

14. Diana, J. \& Lehuen, A. NKT cells: friend or foe during viral infections? Eur. J. Immunol. 39, 3283-3291 (2009).

15. Juno, J.A., Keynan, Y. \& Fowke, K.R. Invariant NKT cells: regulation and function during viral infection. PLOS Pathog. 8, e1002838 (2012).

16. van der Vliet, H.J. et al. Selective decrease in circulating Valpha $24+V$ beta $11+$ NKT cells during HIV type 1 infection. J. Immunol. 168, 1490-1495 (2002).

17. Fernandez, C.S. et al. Peripheral NKT cells in simian immunodeficiency virus-infected macaques. J. Virol. 83, 1617-1624 (2009).

18. van der Vliet, H.J. et al. Cutting edge: rapid recovery of NKT cells upon institution of highly active antiretroviral therapy for HIV-1 infection. J. Immunol. 177, 5775-5778 (2006).

19. Snyder-Cappione, J.E. et al. Lower cytokine secretion ex vivo by natural killer T cells in HIV-infected individuals is associated with higher CD161 expression. AIDS 23, 1965-1970 (2009).

20. Moll, M. et al. Severe functional impairment and elevated PD-1 expression in CD1d-restricted NKT cells retained during chronic HIV-1 infection. Eur. J. Immunol. 39, 902-911 (2009).

21. Roelofs-Haarhuis, K., Wu, X. \& Gleichmann, E. Oral tolerance to nickel requires CD4 + invariant NKT cells for the infectious spread of tolerance and the induction of specific regulatory T cells. J. Immunol. 173, 1043-1050 (2004).

22. Sandberg, J.K. et al. Selective loss of innate CD4(+) V alpha 24 natural killer T cells in human immunodeficiency virus infection. J. Virol. 76, 75287534 (2002).

23. Motsinger, A. et al. CD1d-restricted human natural killer T cells are highly susceptible to human immunodeficiency virus 1 infection. J. Exp. Med. 195, 869-879 (2002).
24. Fernandez, C.S., Kelleher, A.D., Finlayson, R., Godfrey, D.I. \& Kent, S.J. NKT cell depletion in humans during early HIV infection. Immunol. Cell Biol. 92, 578-590 (2014).

25. Josefowicz, S.Z., Lu, L.F. \& Rudensky, A.Y. Regulatory T cells: mechanisms of differentiation and function. Annu. Rev. Immunol. 30, 531-564 (2012).

26. Brenchley, J.M. et al. Microbial translocation is a cause of systemic immune activation in chronic HIV infection. Nat. Med. 12, 1365-1371 (2006).

27. Hunt, P.W. et al. Gut epithelial barrier dysfunction and innate immune activation predict mortality in treated HIV infection. J. Infect. Dis. 210, 1228-1238 (2014).

28. Nockher, W.A., Bergmann, L. \& Scherberich, J.E. Increased soluble CD14 serum levels and altered CD14 expression of peripheral blood monocytes in HIV-infected patients. Clin. Exp. Immunol. 98, 369-374 (1994).

29. Pelsers, M.M. et al. Intestinal-type and liver-type fatty acid-binding protein in the intestine. Tissue distribution and clinical utility. Clin. Biochem. 36, 529-535 (2003).

30. Lozupone, C.A. et al. HIV-induced alteration in gut microbiota: driving factors, consequences, and effects of antiretroviral therapy. Gut Microbes. 5, 562-570 (2014).

31. Dillon, S.M. et al. An altered intestinal mucosal microbiome in HIV-1 infection is associated with mucosal and systemic immune activation and endotoxemia. Mucosal Immunol. 7, 983-994 (2014).

32. Ibarrondo, F.J. et al. Preferential depletion of gut CD4-expressing iNKT cells contributes to systemic immune activation in HIV-1 infection. Mucosal Immunol. 6, 591-600 (2013).

33. Chan, A.C. et al. Ex vivo analysis of human natural killer $T$ cells demonstrates heterogeneity between tissues and within established CD4(+) and CD4( - ) subsets. Clin. Exp. Immunol. 172, 129-137 (2013).

34. Gumperz, J.E., Miyake, S., Yamamura, T. \& Brenner, M.B. Functionally distinct subsets of CD1d-restricted natural killer T cells revealed by CD1d tetramer staining. J. Exp. Med. 195, 625-636 (2002).

35. Lee, P.T., Benlagha, K., Teyton, L. \& Bendelac, A. Distinct functional lineages of human V(alpha)24 natural killer T cells. J. Exp. Med. 195, 637-641 (2002).

36. Rout, N. et al. Loss of effector and anti-inflammatory natural killer $T$ lymphocyte function in pathogenic simian immunodeficiency virus infection. PLoS Pathog. 8, e1002928 (2012).

37. Madsen, K.L. et al. Interleukin-10 gene-deficient mice develop a primary intestinal permeability defect in response to enteric microflora. Inflamm. Bowel Dis. 5, 262-270 (1999).

38. Nowak, P. et al. Gut microbiota diversity predicts immune status in HIV-1 infection. AIDS 29, 2409-2418 (2015).

39. Loh, L., Ivarsson, M.A., Michaelsson, J., Sandberg, J.K. \& Nixon, D.F. Invariant natural killer Tcells developing in the human fetus accumulate and mature in the small intestine. Mucosal Immunol. 7, 1233-1243 (2014).

40. Gadola, S.D., Dulphy, N., Salio, M. \& Cerundolo, V. Valpha24-JalphaQindependent, CD1d-restricted recognition of alpha-galactosylceramide by human CD4(+) and CD8alphabeta(+) T lymphocytes. J. Immunol. 168, 5514-5520 (2002).

41. Caporaso, J.G. et al. QIIME allows analysis of high-throughput community sequencing data. Nat Methods 7, 335-336 (2010).

42. Huang, Y. et al. A simple LC-MS/MS method for determination of kynurenine and tryptophan concentrations in human plasma from HIV-infected patients. Bioanalysis 5, 1397-1407 (2013).

This work is licensed under a Creative Commons Attribution-NonCommercial-NoDerivs 4.0 International License. The images or other third party material in this article are included in the article's Creative Commons license, unless indicated otherwise in the credit line; if the material is not included under the Creative Commons license, users will need to obtain permission from the license holder to reproduce the material. To view a copy of this license, visit http://creativecommons.org/licenses/by-nc-nd/4.0/ 